UWThPh-2002-14

October 2002

\title{
On the Orthogonality of Independently Propagating States as Occurring in the Lee-Oehme-Yang Theory
}

\author{
G.V. Dass \\ Physics Department, Indian Institute of Technology \\ Powai, Bombay 400076, India \\ W. Grimus \\ Institut für Theoretische Physik, Universität Wien \\ Boltzmanngasse 5, A-1090 Wien, Austria
}

\begin{abstract}
We generalize a theorem by Khalfin, originally derived for the states $\left|F_{1}\right\rangle=$ $\left|M^{0}\right\rangle,\left|F_{2}\right\rangle=\left|\bar{M}^{0}\right\rangle$, where $M^{0}$ is a neutral flavoured meson (e.g., $K^{0}$ or $B_{d}^{0}$ ), by assuming CPT invariance. Dispensing with CPT invariance and allowing for an arbitrary pair of orthogonal states $\left|F_{1,2}\right\rangle$, we show that any linear combinations $\left|P_{a}\right\rangle=p_{a}\left|F_{1}\right\rangle+q_{a}\left|F_{2}\right\rangle$ and $\left|P_{b}\right\rangle=p_{b}\left|F_{1}\right\rangle-q_{b}\left|F_{2}\right\rangle$, if postulated to be independently propagating in time, as in the Lee-Oehme-Yang Theory, must be mutually orthogonal. This implies a reciprocity relation: equality of the probabilities of the transitions $\left|F_{1}\right\rangle \leftrightarrow\left|F_{2}\right\rangle$. Also implied is another relation involving the coefficients $p_{a, b}, q_{a, b}$, which can be interpreted as $\operatorname{Im} \theta=$ 0 , where $\theta$ is the rephasing-invariant parameter describing CPT violation in $M^{0} \bar{M}^{0}$ mixing for Khalfin's choice of $\left|F_{1,2}\right\rangle$. The states $\left|F_{1,2}\right\rangle$ of our theorem need not form a particle-antiparticle pair, nor even be restricted to particle physics.
\end{abstract}

PACS numbers: 03.65.-w, 11.30.Er 
The complex formed by a neutral meson $M^{0}$ (i.e., $\left.K^{0}, D^{0}, B_{d}^{0}, B_{s}^{0}\right)$ and its antiparticle $\bar{M}^{0}$ is a very important testing ground for discrete symmetries and physics beyond the Standard Model. Its theoretical treatment relies heavily on the Weisskopf-Wigner approximation [1] and the Lee-Oehme-Yang (LOY) Theory [2] (for reviews see, for instance, Refs. [3-5]). Central to this approximate theory are the linear combinations

$$
\begin{aligned}
\left|M_{H}\right\rangle & =p_{H}\left|M^{0}\right\rangle+q_{H}\left|\bar{M}^{0}\right\rangle, \quad\left|p_{H}\right|^{2}+\left|q_{H}\right|^{2}=1, \\
\left|M_{L}\right\rangle & =p_{L}\left|M^{0}\right\rangle-q_{L}\left|\bar{M}^{0}\right\rangle, \quad\left|p_{L}\right|^{2}+\left|q_{L}\right|^{2}=1,
\end{aligned}
$$

with complex constants $p_{H, L}$ and $q_{H, L}$, which propagate independently in time. This property is also called "lack of vacuum regeneration". The subscripts $H$ and $L$ refer to heavy and light, respectively. By starting with the additional assumption of

$$
\text { CPT invariance in } M^{0} \bar{M}^{0} \text { mixing: } \frac{q_{H}}{p_{H}}=\frac{q_{L}}{p_{L}},
$$

Khalfin demonstrated [6,7] that the independent propagation of $\left|M_{H}\right\rangle,\left|M_{L}\right\rangle$ and positivity of the spectrum of the total Hamiltonian governing the time evolution of $\left|M^{0}\right\rangle,\left|\bar{M}^{0}\right\rangle$ and of the decay states $|f\rangle$ leads to $\left\langle M_{H} \mid M_{L}\right\rangle=0$ ("Main Theorem, Part (A)" in Ref. [6]). This result is apparently at odds with the experimental finding of $\mathrm{CP}$ violation in $K^{0} \bar{K}^{0}$ mixing [8,9], which is equivalent to $\left\langle K_{L} \mid K_{S}\right\rangle \neq 0$, if CPT invariance holds in $K^{0} \bar{K}^{0}$ mixing. Note that in the kaon system we make the identification $\left|K_{L}\right\rangle=\left|M_{H}\right\rangle,\left|K_{S}\right\rangle=\left|M_{L}\right\rangle$. Khalfin's purpose was to demonstrate forcefully, on the basis of general principles, the inadequacy of the LOY Theory as a good theory of CP violation. While the mathematical content of his theorem remains true, his claim of a resulting large "new CP violation effect" was subsequently refuted in Refs. [10,11]; we shall return to this point later.

In this paper, we generalize Khalfin's Theorem by dropping the requirement of CPT invariance. ${ }^{1}$ It is interesting to relax this requirement in view of the need of improvement of present data for many of the tests of CPT invariance [13]. Because of our generalization, we go beyond the choice of $\left|F_{1,2}\right\rangle$ being a particle-antiparticle pair, though, at present, this choice seems to be the only one with an experimental application in particle physics. ${ }^{2}$

We consider a pair of orthonormal electrically neutral states $\left|F_{1,2}\right\rangle$, in analogy to $\left|M^{0}\right\rangle$ and $\left|\bar{M}^{0}\right\rangle$, and states $|f\rangle$ orthogonal to $\left|F_{1,2}\right\rangle$. Then the time evolution of the states $\left|F_{1,2}\right\rangle$ is, most generally, expressed as

$$
\left|F_{j}(t)\right\rangle=\sum_{k=1,2}\left|F_{k}\right\rangle A_{k j}(t)+\sum_{f} c_{f j}(t)|f\rangle \quad \text { with } \quad A_{k j}(t)=\left\langle F_{k}\left|e^{-i H t}\right| F_{j}\right\rangle \quad \forall t \geq 0 .
$$

Now we want to be more specific about the time evolution (3). We postulate the existence of two linearly independent superpositions $\left|P_{a, b}\right\rangle$ of $\left|F_{1,2}\right\rangle$, defined by

\footnotetext{
${ }^{1}$ In an unpublished report [12], quoted as Ref. [30] in his paper [6], Khalfin relaxed the assumption of CPT invariance.

${ }^{2}$ Thus our theorem is not restricted to particle physics. See remarks at the end of Ref. [10] for its applicability in the study of communicating metastable states in atomic physics.
} 


$$
\begin{aligned}
& \left|P_{a}\right\rangle=p_{a}\left|F_{1}\right\rangle+q_{a}\left|F_{2}\right\rangle, \quad\left|p_{a}\right|^{2}+\left|q_{a}\right|^{2}=1, \\
& \left|P_{b}\right\rangle=p_{b}\left|F_{1}\right\rangle-q_{b}\left|F_{2}\right\rangle, \quad\left|p_{b}\right|^{2}+\left|q_{b}\right|^{2}=1
\end{aligned}
$$

where $p_{a, b}$ and $q_{a, b}$ are complex constants, such that these states propagate independently in time. Eq. (4) is the analogue of Eq. (1). This means that the time evolution of $\left|P_{a, b}\right\rangle$ is described for $t \geq 0$ by

$$
\begin{aligned}
& \left|P_{a}\right\rangle \stackrel{t}{\rightarrow} \lambda_{a}(t)\left|P_{a}\right\rangle+\sum_{f} c_{f a}(t)|f\rangle, \\
& \left|P_{b}\right\rangle \stackrel{t}{\rightarrow} \lambda_{b}(t)\left|P_{b}\right\rangle+\sum_{f} c_{f b}(t)|f\rangle,
\end{aligned}
$$

where $\lambda_{a, b}$ are suitable propagation functions and the $|f\rangle$ terms in this equation are directly related to those in Eq. (3). The main point in Eq. (5) is that there are no transitions $\left|P_{a}\right\rangle \stackrel{t}{\rightarrow}\left|P_{b}\right\rangle$ or $\left|P_{b}\right\rangle \stackrel{t}{\rightarrow}\left|P_{a}\right\rangle$.

Now we formulate our theorem which generalizes the "Main Theorem, Part (A)" of Khalfin in Ref. [6].

Theorem: If the spectrum of the Hamiltonian $H$ is positive (or, more generally, bounded from below), then the two independently propagating states $\left|P_{a, b}\right\rangle$ are mutually orthogonal, i.e., $\left\langle P_{a} \mid P_{b}\right\rangle=0$.

Proof: The time evolution as expressed by Eq. (3) is connected with Eq. (5) via

$$
\operatorname{diag}\left(\lambda_{a}(t), \lambda_{b}(t)\right) \equiv B(t)=R^{-1} A(t) R \quad \text { with } \quad R=\left(\begin{array}{cc}
p_{a} & p_{b} \\
q_{a} & -q_{b}
\end{array}\right) .
$$

Now, the independent propagation of the states $\left|P_{a, b}\right\rangle$ is formulated as [14]

$$
\begin{aligned}
& B_{21}=\left\{\left(A_{11}-A_{22}\right) p_{a} q_{a}+A_{12} q_{a}^{2}-A_{21} p_{a}^{2}\right\} / D=0, \\
& B_{12}=\left\{\left(A_{11}-A_{22}\right) p_{b} q_{b}-A_{12} q_{b}^{2}+A_{21} p_{b}^{2}\right\} / D=0,
\end{aligned}
$$

where $D=p_{a} q_{b}+p_{b} q_{a}=-\operatorname{det} R$. From these equations we derive [14]

$$
\begin{aligned}
A_{12} & =\frac{p_{a} p_{b}}{q_{a} q_{b}} A_{21}, \\
A_{11}-A_{22} & =\left(\frac{p_{a}}{q_{a}}-\frac{p_{b}}{q_{b}}\right) A_{21} .
\end{aligned}
$$

Following Khalfin [6] (see also Refs. [10,15]), we introduce the expansions

$$
\left|F_{j}\right\rangle=\int_{0}^{\infty} \mathrm{d} m \sum_{\alpha} d_{j \alpha}(m)\left|\phi_{\alpha}(m)\right\rangle \quad(j=1,2)
$$

over the complete set of orthonormal eigenfunctions $\left|\phi_{\alpha}(m)\right\rangle$ of the full Hamiltonian $H$ :

$$
H\left|\phi_{\alpha}(m)\right\rangle=m\left|\phi_{\alpha}(m)\right\rangle \quad \text { with } \quad\left\langle\phi_{\alpha}(m) \mid \phi_{\beta}\left(m^{\prime}\right)\right\rangle=\delta_{\alpha \beta} \delta\left(m-m^{\prime}\right) ;
$$

the $m$-integration is over the non-negative spectrum of $H$. The matrix elements $A_{k j}(t)$ of the time evolution (3) are expressed as 


$$
A_{k j}(t)=\int_{0}^{\infty} \mathrm{d} m e^{-i m t} C_{k j}(m) \quad \text { with } \quad C_{k j}(m)=\sum_{\alpha} d_{k \alpha}^{*}(m) d_{j \alpha}(m) .
$$

Then, Eq. (8) means

$$
\int_{0}^{\infty} \mathrm{d} m e^{-i m t}\left[q_{a} q_{b} C_{12}(m)-p_{a} p_{b} C_{21}(m)\right]=0 \quad \forall t \geq 0 .
$$

We would now like to infer from this equation that the integrand itself is zero. To make this conclusion we need to know that the integral is zero also for negative times $t$. In Refs. $[6,10,15]$ an analyticity argument is used to justify this conclusion; however, the details of this argument are not worked out there. In the appendix we present a full proof that from Eq. (13) it follows indeed that the integral is zero for all times $t$ and that, consequently, the integrand is zero as well. ${ }^{3}$

If the function $C_{12}(m)=C_{21}^{*}(m)$ is identically zero, the mutually orthogonal states $\left|F_{1,2}\right\rangle$ themselves propagate independently in time, because in this case we have $A_{12}(t)=A_{21}(t)=$ 0 for all $t \geq 0$. Let us assume now that $C_{12}(m)$ is not identically zero. We choose a value $m_{0}$ such that $C_{12}\left(m_{0}\right) \neq 0$. Then from the vanishing of the integrand in Eq. (13) we obtain

$$
\frac{p_{a} p_{b}}{q_{a} q_{b}}=\frac{C_{12}\left(m_{0}\right)}{C_{21}\left(m_{0}\right)}
$$

Along the same lines, we derive from Eq. (9) that

$$
C_{11}\left(m_{0}\right)-C_{22}\left(m_{0}\right)=\left(\frac{p_{a}}{q_{a}}-\frac{p_{b}}{q_{b}}\right) C_{21}\left(m_{0}\right) .
$$

It is useful to define the quantities

$$
\rho_{a}=\frac{p_{a}}{q_{a}} e^{-i \gamma}, \rho_{b}=\frac{p_{b}}{q_{b}} e^{-i \gamma} \quad \text { with } \quad C_{12}\left(m_{0}\right)=\left|C_{12}\left(m_{0}\right)\right| e^{i \gamma} .
$$

Note that $\rho_{a, b}$ are invariant under the rephasings $\left|F_{j}\right\rangle \rightarrow\left|F_{j}^{\prime}\right\rangle \equiv e^{i \delta_{j}}\left|F_{j}\right\rangle$ of the states we started with (for a thorough discussion of rephasings in the $M^{0} \bar{M}^{0}$ system, see Ref. [4]). Then, Eq. (14) can be rewritten as

$$
\rho_{a} \rho_{b}=1
$$

The analogous rewriting of Eq. (15) yields

$$
\rho_{a}-\rho_{b}=\frac{C_{11}\left(m_{0}\right)-C_{22}\left(m_{0}\right)}{\left|C_{12}\left(m_{0}\right)\right|} .
$$

\footnotetext{
${ }^{3}$ Note that it would be reasonable to assume that the integrand - as a product of square-integrable functions - is absolutely integrable. However, because of the mathematical intricacies involved in the proof presented in the appendix, we seem to be forced to assume that the integrand in Eq. (13) is square-integrable.
} 
The right-hand side of this equation is real but its value is unknown. Therefore, it is not useful for constraining $\rho_{a, b}$. However, its imaginary part gives

$$
\operatorname{Im} \rho_{a}=\operatorname{Im} \rho_{b}
$$

A little algebra shows that Eqs. (17) and (19) together imply

$$
\operatorname{Im} \rho_{a}=\operatorname{Im} \rho_{b}=0 .
$$

In other words, we finally have

$$
\rho_{a, b} \in \mathbb{R} \quad \text { and } \quad \rho_{a} \rho_{b}=1
$$

Now we consider the overlap between $\left|P_{a}\right\rangle$ and $\left|P_{b}\right\rangle$ :

$$
\left\langle P_{a} \mid P_{b}\right\rangle=p_{a}^{*} p_{b}-q_{a}^{*} q_{b}=q_{a}^{*} q_{b}\left(\rho_{a}^{*} \rho_{b}-1\right) .
$$

Using Eq. (21), we find $\left\langle P_{a} \mid P_{b}\right\rangle=0$, Q.E.D.

We mention two corollaries of our theorem. Firstly, Eq. (14), when used in Eq. (8), directly gives the reciprocity relation $[6,7]$

$$
\left|A_{12}(t)\right|=\left|A_{21}(t)\right|
$$

for the transitions $\left|F_{1}\right\rangle \leftrightarrow\left|F_{2}\right\rangle$; this T-invariance relation is general, no assumptions about the nature of the states $\left|F_{1,2}\right\rangle$ or about symmetries have been made. ${ }^{4}$ For the $M^{0} \bar{M}^{0}$ system, this $\mathrm{T}$ invariance in mixing [16] is usually stated in the form [4]

$$
\left|\frac{q}{p}\right|=1 \quad \text { with } \quad \frac{q}{p}=\sqrt{\frac{q_{a} q_{b}}{p_{a} p_{b}}} .
$$

For the second corollary, we define the quantity

$$
\theta=\frac{\left(q_{a} / p_{a}\right)-\left(q_{b} / p_{b}\right)}{\left(q_{a} / p_{a}\right)+\left(q_{b} / p_{b}\right)}=\frac{\rho_{b}-\rho_{a}}{\rho_{b}+\rho_{a}}
$$

Then, Eq. (20) gives

$$
\operatorname{Im} \theta=0 .
$$

Eqs. (24) and (26) are already known $[4,17]$ to be equivalent to the vanishing of $\left\langle P_{a} \mid P_{b}\right\rangle$ for the particular choice $\left|F_{1}\right\rangle=\left|M^{0}\right\rangle,\left|F_{2}\right\rangle=\left|\bar{M}^{0}\right\rangle$. Our derivation is more general; the $\left|F_{1,2}\right\rangle$ need not form a particle-antiparticle pair. ${ }^{5}$ We want to stress that, if we specialize to the

\footnotetext{
${ }^{4}$ That the relation (23) does not require CPT invariance was already pointed out in Ref. [15].

${ }^{5}$ Note that $\left\langle P_{a} \mid P_{b}\right\rangle=0$ means that the effective Hamiltonian matrix in the subspace generated by $\left|F_{1,2}\right\rangle$ is a normal matrix [4].
} 
$M^{0} \bar{M}^{0}$ system, full CPT invariance in mixing (viz. $\theta=0[4,16,18]$ ) is not required by the theorem; just Eq. (26) is required.

Concentrating on the $K^{0} \bar{K}^{0}$ system, we want to compare the requirement $\left\langle K_{L} \mid K_{S}\right\rangle=0$ of our theorem with available experimental data. Using data on various kaon decay channels and allowing for violations of the discrete symmetries CPT, CP and T and of the $\Delta S=\Delta Q$ rule, the evaluation of the Bell-Steinberger unitarity relation by the CPLEAR Collaboration [9] gives the result ${ }^{6} 1-|q / p|=(3.30 \pm 0.05) \times 10^{-3}$. This result ${ }^{7}$ continues to be far from zero even if the assumption of the saturation of the unitarity relation by known channels is relaxed within the limits allowed by the experimental errors on the decay rates. The Bell-Steinberger unitarity relation is a consequence of the LOY Theory and the WeisskopfWigner approximation. Thus, the CPLEAR result of non-zero $1-|q / p|$, which leads to a non-vanishing overlap $\left\langle K_{L} \mid K_{S}\right\rangle$, is in contradiction to Khalfin's Theorem and its extension proven in the present paper. On the other hand, $\operatorname{Re} \theta$ and $\operatorname{Im} \theta$ are both consistent with zero $[9,19]$.

Though outside the sope of the paper, one might ask the question "What goes wrong when the mathematically correct theorem of Khalfin is applied to the $M^{0} \bar{M}^{0}$ system?" The answer was clearly given in Refs. [10,11,15]: The independent propagation of $\left|M_{H}\right\rangle$ and $\left|M_{L}\right\rangle$ is not strictly true for all times. There is indeed vacuum regeneration to a certain degree and one might nourish the hope that future experiments provide some clue to the size of this effect. However, the "new CP violation effect", predicted by Khalfin [6,7] on the basis of this vacuum regeneration, was an overestimate by many orders of magnitude [10,11].

In summary, starting with two suitable states $\left|F_{1,2}\right\rangle$ which mix to form two independently propagating states $\left|P_{a, b}\right\rangle$ as occurring in the LOY Theory, we have shown the vanishing of the overlap of $\left|P_{a, b}\right\rangle$, i.e., $\left\langle P_{a} \mid P_{b}\right\rangle=0$. We also find the reciprocal transitions $\left|F_{1}\right\rangle \leftrightarrow$ $\left|F_{2}\right\rangle$ to be equally strong, viz. $\mathrm{T}$ invariance in mixing. The restriction to $\left|F_{1,2}\right\rangle$ forming a particle-antiparticle pair is not required, nor the assumption of CPT invariance in the particle-antiparticle case. However, if $\left|F_{1,2}\right\rangle$ do form a particle-antiparticle pair, partial CPT invariance is also required: The imaginary part of the CPT-violating parameter $\theta$ must be zero, whereas the real part of $\theta$ is allowed by the theorem to be non-zero.

\section{ACKNOWLEDGMENTS}

We thank P.K. Kabir and L. Lavoura for important communications.

\footnotetext{
${ }^{6}$ Note that the quantity $1-|q / p|$ is called $2 \operatorname{Re} \varepsilon$ in the CPLEAR paper; see Ref. [21] for a comparison with the CPLEAR notation.

${ }^{7}$ The same result, with a much less compelling accuracy $1-|q / p|=(3.1 \pm 0.7 \pm 0.5) \times 10^{-3}$, was recently obtained by a "direct observation" of $\mathrm{T}$ non-invariance by the CPLEAR Collaboration $[8,19]$; however, this result for $|q / p|$ assumed CPT invariance for the amplitudes of semileptonic neutral kaon decays. A completely assumption-independent "direct measurement" of $|q / p|$ is not available at present; see Ref. [20] and references cited therein.
} 


\section{APPENDIX}

We now present a proof that from Eq. (13) it follows that the function

$$
f(m) \equiv q_{a} q_{b} C_{12}(m)-p_{a} p_{b} C_{21}(m)
$$

must be zero.

Lemma: Let $f$ be a square-integrable function over the real numbers, which is zero on the negative real axis. Defining a function $\hat{f}$ over all real numbers by

$$
\hat{f}(t)=\int_{0}^{\infty} \mathrm{d} m e^{-i t m} f(m)
$$

and assuming that $\hat{f}(t)=0 \forall t \geq 0$, it follows that $\hat{f}(t)=0$ for negative $t$ as well. Consequently, the function $f$ itself is zero.

Proof: The function $\hat{f}(t)$ defined for all real $t$ can be extended to complex numbers $z=t-i \eta$ $(\eta>0)$ in the lower complex half plane, where $\hat{f}(z)$ is an analytic function. Cauchy's Theorem allows us to write

$$
-\frac{1}{2 \pi i} \int_{-\infty}^{\infty} \mathrm{d} u \frac{\hat{f}(u)}{u-z}=\hat{f}(z) \equiv \int_{0}^{\infty} \mathrm{d} m e^{-i z m} f(m) .
$$

Since $f$ is square-integrable, i.e., $f \in L^{2}(\mathbb{R})$, the same holds for $\hat{f}$. Actually, since $f$ is zero on the negative real axis, this function belongs to the class of Hardy functions, for which relation (A2) is common usage (see, for instance, Ref. [22]). Note that the integral on the left-hand side of Eq. (A2) is well-defined since its integrand-as a product of $L^{2}$ functionsis absolutely integrable, i.e., an element of $L^{1}(\mathbb{R})$. Now we assume that $\operatorname{Re} z=t>0$ and take the limit $\operatorname{Im} z=-\eta \uparrow 0$. Then the right-hand side of Eq. (A2) becomes 0 and we arrive at

$$
\int_{0}^{\infty} \mathrm{d} u \frac{g(u)}{u+t}=0 \quad \forall t>0
$$

where we have defined $g(u)=\hat{f}(-u) \forall u \geq 0$. Furthermore, from this equation we derive

$$
\frac{1}{t-1}\left(\int_{0}^{\infty} \mathrm{d} u \frac{g(u)}{u+1}-\int_{0}^{\infty} \mathrm{d} u \frac{g(u)}{u+t}\right)=\int_{0}^{\infty} \mathrm{d} u \frac{g(u)}{(u+1)(u+t)}=0 \quad \forall t \neq 1 .
$$

Because of the uniform convergence of the integral (A4), we are allowed to drop the requirement $t \neq 1$ and the second relation in Eq. (A4) holds thus for all positive $t$. Now we define $G(u)=g(u) /(u+1)$ for $u \geq 0$, which is an absolutely integrable function in $u$. Then, Eq. (A4) is reformulated as

$$
\int_{0}^{\infty} \mathrm{d} u G(u) \int_{0}^{\infty} \mathrm{d} s e^{-s(u+t)}=\int_{0}^{\infty} \mathrm{d} s e^{-t s} \int_{0}^{\infty} \mathrm{d} u e^{-s u} G(u)=(\mathcal{L} \mathcal{L} G)(t)=0
$$


where $\mathcal{L}$ denotes the Laplace transform with integration interval from zero to infinity. ${ }^{8}$ It is easy to argue by using the Theorem of Fubini that, because $G$ is absolutely integrable in $u$, the exchange of the succession of integrations in Eq. (A5) is a correct operation. If the Laplace transform of a function is zero, then the function itself is zero (see, for instance, Ref. [23]). Thus we have proved that $G$ is zero, therefore, also $\hat{f}=0$ and, finally, we find $f=0$, Q.E.D.

${ }^{8}$ One of us (W.G.) is grateful to H. Neufeld for suggesting the use of the Laplace transform. 


\section{REFERENCES}

[1] V. Weisskopf and E. Wigner, Z. Phys. 63, 54 (1930); ibid. 65, 18 (1930).

[2] T. D. Lee, R. Oehme, and C. N. Yang, Phys. Rev. 106, 340 (1957).

[3] P. K. Kabir, The CP Puzzle (Academic Press, London and New York, 1968).

[4] G. C. Branco, L. Lavoura, and J.P. Silva, CP Violation (Oxford University Press, Oxford, 1999).

[5] I. I. Bigi and A. I. Sanda, CP Violation (Cambridge University Press, Cambridge, 2000).

[6] L. A. Khalfin, Foundations of Physics 27, 1549 (1997).

[7] L. A. Khalfin, "New Results on the CP-violation Problem", preprint CPT, The University of Texas at Austin, DOE-ER 40200-211 (1990); "A New CP-violation effect and a new possibility for investigation of $K_{S}, K_{L}\left(K^{0}, \bar{K}^{0}\right)$ decay modes", ibid., DOE-ER 40200-247 (1991).

[8] J. H. Christenson, J. W. Cronin, V. L. Fitch, and R. Turlay, Phys. Rev. Lett. 13, 138 (1964); D. Dorfan et al., Phys. Rev. Lett. 19, 987 (1967); S. Bennett et al., Phys. Rev. Lett. 19, 993 (1967); CPLEAR Collaboration, A. Angelopoulos et al., Phys. Lett B 444, 43 and 52 (1998).

[9] CPLEAR Collaboration, A. Apostolakis et al., Phys. Lett. B 456, 297 (1999).

[10] C. B. Chiu and E. C. G. Sudarshan, Phys. Rev. D 42, 3712 (1990).

[11] M. Nowakowski, Int. J. Mod. Phys. A 14, 589 (1999).

[12] L. A. Khalfin, "An exact theory of the CP-violation problem within CPT-noninvariant quantum theory", 1994, unpublished.

[13] K. Hagiwara et al., Particle Data Group, Phys. Rev. D 66, 010001 (2002).

[14] G. V. Dass and W. Grimus, Phys. Lett. B 521, 267 (2001).

[15] P. K. Kabir and A. N. Mitra, Phys. Rev. D 52, 526 (1995).

[16] P. K. Kabir, Phys. Rev. D 2, 540 (1970); A. Aharony, Lett. Nuovo Cimento 3, 791 (1970).

[17] L. Lavoura, Annals Phys. 207, 428 (1991).

[18] P. K. Kabir, in Springer Tracts in Modern Physics 52, 91 (1970).

[19] CPLEAR Collaboration, A. Angelopoulos et al., Eur. Phys. J. C 22, 55 (2001).

[20] G. V. Dass, Mod. Phys. Lett. A 16, 9 (2001).

[21] G. V. Dass and W. Grimus, hep-ph/0203043.

[22] H. Dym and H. P. McKean, Fourier Series and Integrals (Academic Press, New York and London, 1972).

[23] J. E. Marsden and M. J. Hoffman, Basic Complex Analysis (W. H. Freeman and Company, New York, 1987). 